\title{
OPTIMIZATION OF RUNNING STRATEGIES BASED ON ANAEROBIC ENERGY AND VARIATIONS OF VELOCITY *
}

\author{
AMANDINE AFTALION ${ }^{\dagger}$ AND J. FRÉDÉRIC BONNANS A $^{\ddagger}$
}

\begin{abstract}
We present new models, numerical simulations and rigorous analysis for the optimization of the velocity in a race. In a seminal paper, Keller [11, 12] explained how a runner should determine his speed in order to run a given distance in the shortest time. We extend this analysis, based on the equation of motion and aerobic energy, to include a balance of anaerobic energy (or accumulated oxygen deficit) and an energy recreation term when the speed decreases. We also take into account that when the anaerobic energy gets too low, the oxygen uptake cannot be maintained to its maximal value. Our main results are that constant speed is not optimal, that negative splitting of the race (running the second half faster than the first one) is a good strategy and variations of velocity is energetically the best strategy. Using optimal control theory, we obtain a proof of Keller's optimal race, and relate the problem to a relaxed formulation, where the propulsive force represents a probability distribution rather than a function of time. Our analysis leads us to introduce a bound on the variations of the propulsive force to obtain a more realistic model which displays oscillations of the velocity. Our numerical simulations qualitatively reproduce quite well physiological measurements on real runners. We show how, by optimizing over a period, we recover these oscillations of speed. We point out that our numerical simulations provide in particular the exact instantaneous anaerobic energy used in the exercise.
\end{abstract}

Key words. Running race, anaerobic energy, energy recreation, optimal control, singular arc, state constraint, optimality conditions.

1. Introduction. The issue of optimizing a race given the distance or time to run is a major one in sports competition and training. A pioneering work is that of Keller 12 relying on Newton's law of motion and energy equilibrium. For sufficiently long races $(>291 \mathrm{~m})$, his analysis leads to an optimal run in three parts

1. initial acceleration at maximal force of propulsion,

2. constant speed during the most part of the race,

3. final small part with constant energy equal to zero.

This analysis has some drawbacks and does not describe well some physiological properties, in particular the last part: it is unbelievable that a runner can go on running with zero energy. Additionally, Keller does not prove that the optimal race has exactly these three parts like this, but rather makes up a race with these three optimal pieces together. Some authors [1, 13, 24] have tried to improve this model, in particular the last part of the race, but still relying on the same strategy and mathematical arguments, leading to an almost constant speed. The idea of the constant speed is a controversial one. On some marathon runs, the constant speed theory is used to guide runners who can choose the color of balloon they follow which corresponds to a constant speed run. Nevertheless, recent physiological measurements [3] seem to indicate that in order to optimize his run, a runner varies his velocity by an order of $10 \%$. This allows him to recreate anaerobic energy. Other references concerning the optimality of a run include [15, 17, 23. Keller's paper 12 has some interesting ideas that we will rely on to build a more satisfactory model, using additionally the

\footnotetext{
*Preprint: Rapport de Recherche Inria 8446, August 2013. Version of Aug. 12th, 2013

${ }^{\dagger}$ Laboratoire de Mathématiques de Versailles, CNRS UMR 8100, 45 avenue des Etats-Unis, 78035 Versailles Cédex, France; amandine.aftalion@uvsq.fr.

${ }^{\ddagger}$ INRIA-Saclay and Centre de Mathématiques Appliquées, Ecole Polytechnique, 91128 Palaiseau, France; email: Frederic.Bonnans@inria.fr.
} 
hydraulic analogy and physiological improvements described in [16. Nevertheless, the formula of [16] rely on averaged values while we want to make instantaneous energy balance taking into account optimal control theory. We aim at fully accounting for measurements of $[3]$.

Human energy can be split into aerobic energy called $e_{a e}$, which is the energy provided by oxygen consumption, and anaerobic energy $e_{a n}$, which is provided by glycogen and lactate. A very good review on different types of modeling can be found in [16] and a more general reference is [2. In this paper, we will focus on improving the model of Keller [12. At first sight, one may believe that Keller's equations only only with aerobic energy: he speaks of oxygen supplies. In fact, as we will show below, we believe that it well describes the accumulated oxygen deficit: $e_{a n}^{0}-e_{a n}$, here $e_{a n}^{0}$ is the value at $t=0$ of $e_{a n}$ the anaerobic energy. This will use the hydraulic analogy introduced by Morton and other authors (see [15, 16]).

We will call $v_{M A}$ the maximal aerobic velocity (it is also called $v_{V O 2 \max }$ ). When one runs below $v_{M A}$, the value of $\dot{V} O 2_{\max }$ (maximal oxygen uptake) has not been reached. The role of the anaerobic energy is to compensate the deficit in $\dot{V} O 2$ which has not reached its maximal value. When one goes above $v_{M A}$, the anaerobic energy has two effects: giving energy to run above $v_{M A}$ and to maintain $\dot{V} O 2$ at its maximal value. This is why when $e_{a n}$ gets too low, $\dot{V} O 2$ cannot be maintained to its maximal value. Next, when one is between $0.8 v_{M A}$ and $v_{M A}$, if the runner varies his velocity, the excess of aerobic energy available due to slowing down allows the runner to fuel its anaerobic supplies.

In our model, we will account for

- the drop in $\dot{V} O 2$ at the end of the race, when the anaerobic supply get too low,

- the use of anaerobic energy at the beginning of the race to compensate the deficit in $\dot{V} O 2$ which does not reach its maximal value instantaneously,

- the fact that negative splitting of the race is better than running at constant speed,

- how varying one's velocity allows to recreate anaerobic energy and thus to run longer or faster.

The aim of what follows is to determine the equation governing the energy $e_{a n}$, or the accumulated oxygen deficit. As a consequence, our simulations provide accurate estimates of the loss of anaerobic energy in a race.

The paper is organized as follows: in section 2, we present our new models together with numerical simulations. In section 3 , we describe our mathematical analysis and proofs while a conclusion is derived in section 4 .

2. Numerical presentation of the models. In this section, we will present our numerical findings for Keller's model and our ideas for improved modeling fitting better the physiological measurements. Our numerical experiments are based on the Bocop toolbox for solving optimal control problems [6]. This software combines a user friendly interface, general Runge-Kutta discretization schemes described in [10, 5, and the numerical resolution of the discretized problem using the nonlinear programming problems solver IPOPT [22.

The aim of what follows is to determine the equation governing the energy $e_{a n}$. This will be coupled with the equation of motion

$$
\frac{d v}{d t}+\frac{v}{\tau}=f(t)
$$


where $t$ is the time, $v(t)$ is the instantaneous velocity, $f(t)$ is the propulsive force and $v / \tau$ is a resistive force per unit mass. The resistive force can be modified as another power of $v$, but we will use this one in the simulations for simplicity. Note that we could take into account a changing altitude, by adding to the right hand side a term of the form $-g \sin \alpha(d)$, where $\alpha(d(t))$ is the slope at distance $d(t)$. We can relate $\sin \alpha(d)$ to $h(d)$, the height of the center of mass of the runner at distance $d$, by

$$
\sin \alpha(d(t))=\frac{h^{\prime}(d)}{1+\left(h^{\prime}(d)\right)^{2}} .
$$

Here, we assume for simplicity that $h$ is constant along the race.

Constraints have to be imposed; the force is controlled by the runner but it cannot exceed a maximal value:

$$
0 \leq f(t) \leq f_{\max }
$$

Then the aim is to optimize $T$ the total time, given $d=\int_{0}^{T} v(t) d t$, with the initial conditions:

$$
v(0)=0, \quad e_{a n}(0)=e_{a n}^{0} \quad \text { under the constraint } e_{a n}(t) \geq 0 .
$$

Let us use the hydraulic analogy to account for Keller's equations and justify our improvements. This hydraulic analogy is described in [15, 16] in order to develop a three parameter critical power model: the equations in [15, 16] are on averaged values of the energy and the power, while we use instantaneous values.

2.1. First model: how Keller's model describes the accumulated oxygen deficit. We assume that the anaerobic energy has finite capacity modeled by a container of height 1 and surface $A_{p}$. When it starts depleting by a height $h$, then the accumulated oxygen deficit is $e_{a n}^{0}-e_{a n}$ and we have

$$
e_{a n}^{0}-e_{a n}=A_{p} h,
$$

where $e_{a n}^{0}=A_{p}$ is the initial supply of anaerobic energy.

We assume that the aerobic energy is of infinite capacity and flows at a maximal rate of $\dot{V} O 2_{\max }$ through $R_{1}$. We refer to Figure 2.1 for an illustration: $O$ is the infinite aerobic contained, $P$ is the finite capacity anaerobic container, $h$ is the height of depletion of the anaerobic container. An important point is the height at which the aerobic container is connected to the anaerobic one. If we assume in this first model that it is connected at height 1 (at the top of the anaerobic container, and not $\varphi$ for the moment as on Figure 2.1), then it means that the aerobic energy always flows at rate $\bar{\sigma}=\dot{V} O 2_{\max }$ and the available flow at the bottom of the anaerobic container, through $T$ is

$$
W=\bar{\sigma}+A_{p} \frac{d h}{d t}=\bar{\sigma}-\frac{d e_{a n}}{d t} .
$$

Since the energy is used at a rate $f v$, where $v$ is the velocity and $f$ is the propulsive force, we have that $W$ is equal to the available work capacity hence to $f v$. This allows us to find the equation governing the evolution of the anaerobic energy

$$
\frac{d e_{a n}}{d t}=\bar{\sigma}-f v .
$$




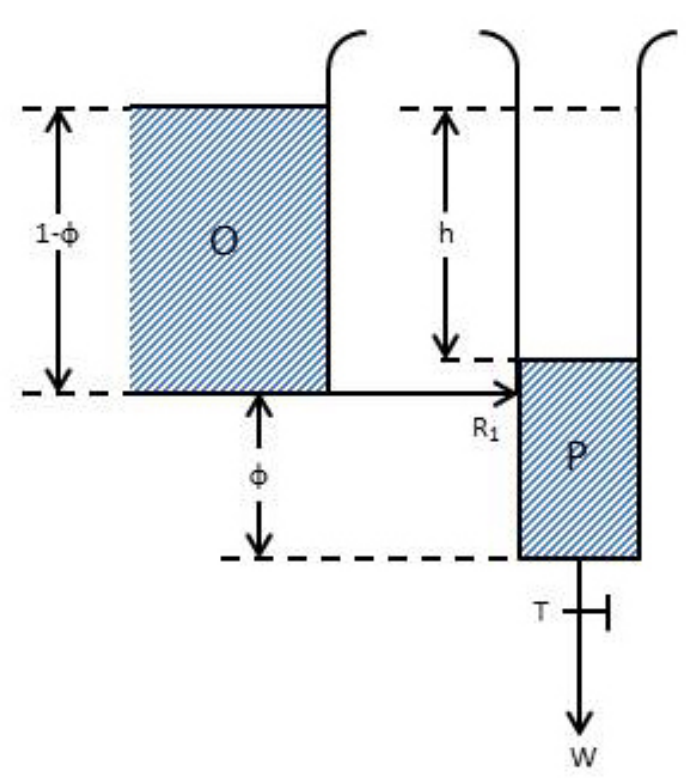

FIG. 2.1. Scheme of the container modeling.

We point out that this is exactly the energy equation studied by Keller, except that we have justified that it models the accumulated oxygen deficit, while Keller describes it as the aerobic energy.

Some improvements are needed for this model to better account for the physiology:

- change the height where the aerobic container is connected,

- take into account that when the energy supply is low, then the flow of energy drops significantly.

Before improving the model, we describe our numerical simulations of (2.1)-(2.2)2.3)-(2.5) using bocop.

We plot, in figure 2.2. the velocity $v$, the force $f$, the accumulated oxygen deficit (AOD) $e_{a n}^{0}-e_{a n}$. We have added $\bar{\sigma}$, though it is constant, just to be consistent with the next figures. We take $\bar{\sigma}=41.56, f_{\max }=9, e_{a n}^{0}=2409$ and $d=800 \mathrm{~m}$. The optimal time is 106.01, and we have 2000 discretization steps, i.e. the time step is close to $0.053 \mathrm{~s}$. We display in figure 3.1 a detailed view of what happens at the end of the race for the AOD and the force.

We observe that the race splits into three parts

- The race starts with a strong acceleration, the velocity increases quickly and the force is at its maximal value,

- for the major part of the race, the force is at an intermediate constant value, the velocity is constant with value close to $7.6 \mathrm{~m} / \mathrm{s}$. We will see that this corresponds to what is called, in the optimal control theory, a singular arc.

- during the last part corresponding to the last two seconds, the force sharply decreases, the energy reaches 0 and then stays at the zero level (AOD is constant equal to $e_{a n}^{0}$ ), and the force slightly increases again; during all that 

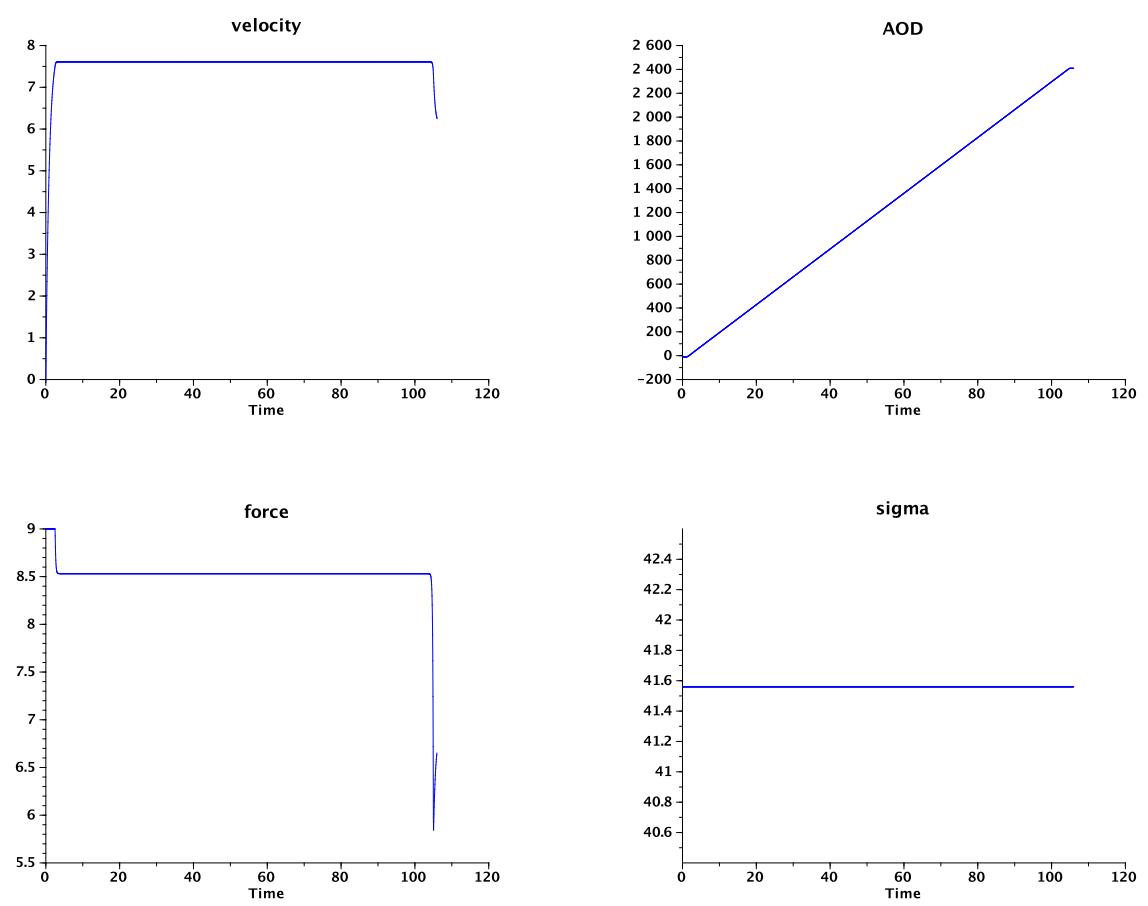

FIG. 2.2. Race problem with Keller's model. Plot of the velocity $v$, the accumulated oxygen deficit $(A O D) e_{a n}^{0}-e_{a n}$, the propulsive force $f$, and $\bar{\sigma}$ vs time.

time, the velocity decreases.

We insist on the fact that this is the first simulation not based on the hypothesis that there are three arcs. Also, we can optimize either on the time to run or the distance to run, where all previous simulations had to fix the time to run and optimize on the distance. Even if this is mathematically equivalent in terms of optimization, fixing the distance requires an extra parameter in the simulations. The next models introduce improvements.

2.2. Second model: improving the initial phase to reach $\dot{V} O 2_{\max }$. We now assume that the aerobic container is connected to the anaerobic container at a height $\varphi \in(0,1)$. This implies that there is an initial phase of the race where the flow from the aerobic container to the anaerobic one is no longer $\bar{\sigma}$, but is proportional to the difference of fluid heights in the containers, so that,

$$
\sigma(h)=\left\{\begin{array}{l}
\bar{\sigma} \frac{h}{1-\varphi} \text { when } h<1-\varphi \\
\bar{\sigma} \text { when } h \geq 1-\varphi
\end{array}\right.
$$

This is illustrated in Figure 2.1. We still have the same balance on total work capacity namely

$$
W=f v=\sigma(h)+A_{p} \frac{d h}{d t} .
$$


So this and 2.4 lead to the following equations for $e_{a n}$ :

$$
\frac{d e_{a n}}{d t}=\lambda \bar{\sigma}\left(e_{a n}^{0}-e_{a n}\right)-f v \quad \text { when } \quad \lambda\left(e_{a n}^{0}-e_{a n}\right)<1,
$$

where $1 / \lambda=A_{p}(1-\varphi)$. Numerically, we expect that $\lambda\left(e_{a n}^{0}-e_{a n}\right)$ reaches 1 in about 20 to 40 seconds, so that we choose $\varphi=0.8$.

In the second phase, when $\lambda\left(e_{a n}^{0}-e_{a n}\right)$ has reached 1 , we are back to equation 2.5, that is

$$
\frac{d e_{a n}}{d t}=\bar{\sigma}-f v \quad \text { when } \quad \lambda\left(e_{a n}^{0}-e_{a n}\right)>1 .
$$

This model accounts, in a more satisfactory way, for the beginning of the race, where $\dot{V} O 2$ does not reach instantaneously its maximal value $\dot{V} O 2_{\max }$.

2.3. Third model: drop in $\dot{V} O 2$ at the end of the race. We want to keep the same initial phases as in the previous model, but take into account that there are limitations when the energy supply is small. It is a very important measurement of 3 that $\dot{V} O 2$ drops in the last part of the race. Morton [16 suggests to write that the work capacity is proportional to $e_{a n}$ when $e_{a n}$ is small. We prefer to assume that $\sigma$ drops (drop in $\dot{V} O 2$ ) when $e_{a n}$ is too small. So we add a last phase to the run: when $e_{a n} / e_{a n}^{0}<e_{c r i t}$, then

$$
\bar{\sigma} \text { is replaced by } \bar{\sigma} \frac{e_{a n}}{e_{a n}^{0} e_{c r i t}} \text {. }
$$

We can choose for instance $e_{\text {crit }}=0.2$. We add the final stage:

$$
\frac{d e_{a n}}{d t}=\bar{\sigma} \frac{e_{a n}}{e_{a n}^{0} e_{c r i t}}-f v \quad \text { when } \quad \frac{e_{a n}}{e_{a n}^{0}}<e_{c r i t} .
$$

The coupling of the 3 equations 2.8$), 2.9 p$ when $\frac{e_{a n}}{e_{a n}^{0}}>e_{c r i t}$ on the one hand, and 2.10 on the other hand, leads to a better running profile. This model is much more satisfactory than Keller's initial model. It takes care of fatigue with a much better physiological description than [1, 13. This model can be summarized as follows

$$
\frac{d e_{a n}}{d t}=\sigma\left(e_{a n}\right)-f v
$$

where

$$
\sigma\left(e_{a n}\right)=\left\{\begin{array}{l}
\bar{\sigma} \frac{e_{a n}}{e_{a n}^{0} e_{c r i t}} \text { if } \frac{e_{a n}}{e_{a n}^{0}}<e_{c r i t} \\
\bar{\sigma} \text { if } \frac{e_{a n}}{e_{a n}^{0}} \geq e_{c r i t} \text { and } \lambda\left(e_{a n}^{0}-e_{a n}\right) \geq 1 \\
\lambda \bar{\sigma}\left(e_{a n}^{0}-e_{a n}\right) \text { if } \lambda\left(e_{a n}^{0}-e_{a n}\right)<1
\end{array}\right.
$$

together with 2.1)-(2.2)-(2.3).

Let us now describe our numerical findings. The results are displayed in Figure 2.3. Since $\sigma$ is not constant, the singular arc has no longer a constant velocity but there is a negative split of the run. Let us be more specific:

- The very first part of the race is still at maximal force with a strong acceleration,

- then the force smoothly decreases to its minimal value at the middle of the race, and so does the velocity. 

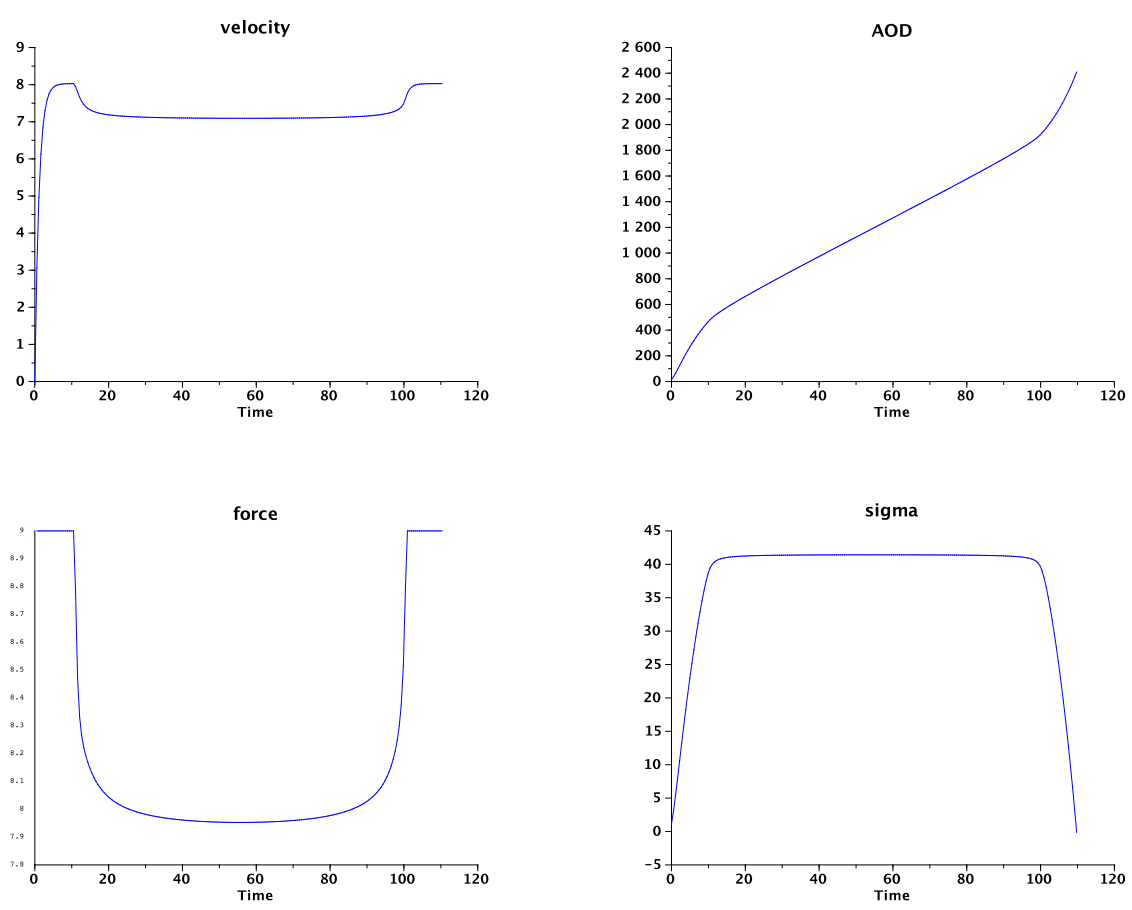

FIG. 2.3. Recreation with variable $\sigma$ : Plot of the velocity $v$, the accumulated oxygen deficit (AOD) $e_{a n}^{0}-e_{a n}$, the propulsive force $f$, and $\sigma$ vs time.

- then the velocity and force smoothly increase again

- the last part of the race is at maximal force, corresponding to the final sprint. The final time is 110.3. Let us point out that depending on the number of computational points, the software indicates a very last part at constant energy, on a couple of points of discretization. The choice of $\sigma\left(e_{a n}\right)$ modifies the intensity of the split of the run. Here, we have chosen a symmetric $\sigma$, but any profile can be entered into the computation.

We now want to take into account the observation of energy recreation when slowing down.

2.4. Fourth model: energy recreation when slowing down. We want to add energy recreation to 2.11, 2.12). Namely, we replace 2.11 by

$$
\frac{d e_{a n}}{d t}=\sigma\left(e_{a n}\right)+\eta(a)-f v
$$

where $a=\frac{d v}{d t}$ is the acceleration and we choose $\eta(a)=c a_{-}^{2}$ where $a_{-}$is the negative part of $a$. In other words,

$$
\eta(a)=0 \text { if } a \geq 0 \text { and } \eta(a)=c|a|^{2} \text { if } a \leq 0 .
$$

When the runner slows down, this recreates anaerobic energy. This dependence on acceleration is motivated by experiments of V.Billat [18].

Because of this new term, the hamiltonian gets non convex, so that by Pontryagin's maximum principle (see our analysis in section 3.3.2 the optimal solution 

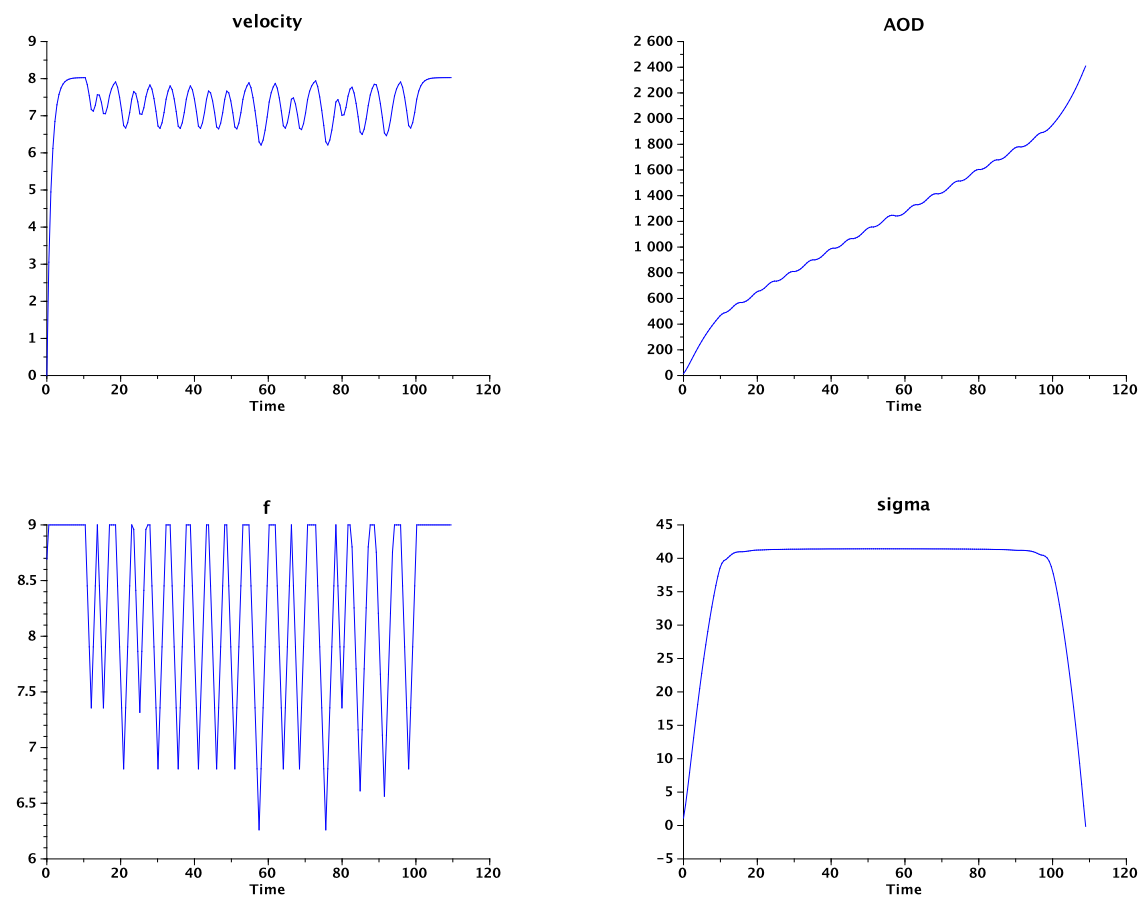

FIG. 2.4. Recreation when slowing down: speed, energy, force, $\sigma$.

oscillates between the maximal and minimal value of the force (i.e. $f_{\max }$ and 0 ). This is in fact to be understood in a relaxed sense, as a probabilty of taking the maximal and minimal values of the force. However, for the runner, the information to vary its propulsive force takes some time to reach the brain so that the runner cannot change his propulsive force instantaneously. We choose to take this into account by bounding the derivative of $f$ :

$$
\left|\frac{d f}{d t}\right| \leq C .
$$

The simulations of (2.1)-(2.2)-(2.3)-(2.12)- 2.13$)$ are illustrated in figure 2.4. We take $C=0.1$ in 2.15) and $c=10$ in 2.14). The optimal time is 109.53 , which is very good.

We see that the force, having a bounded derivative, does not oscillates between its maximal value and 0 , but between its maximal value, and some lower value, the derivative of the force reaching its bounds. Consequently, the velocity oscillates and so does the energy which gets recreated. These oscillation reproduce in a very convincing way the measurements of $[\underline{3}$.

2.5. A periodic pattern. The previous experiments show a behavior of the optimal control which looks, in the time interval $[15,96]$, i.e., except for the initial and final part of the trajectory, close to a periodic one. We have approximately $e_{a n}(15.4)=1845$ and $e_{a n}(95.9)=528$, and so the average decrease per unit time is $e_{d}=16.36$. We observe that over this time interval the speed varies between 6.6 and $7.9 \mathrm{~m} / \mathrm{s}$, and the force varies between 6.8 and 9 . 

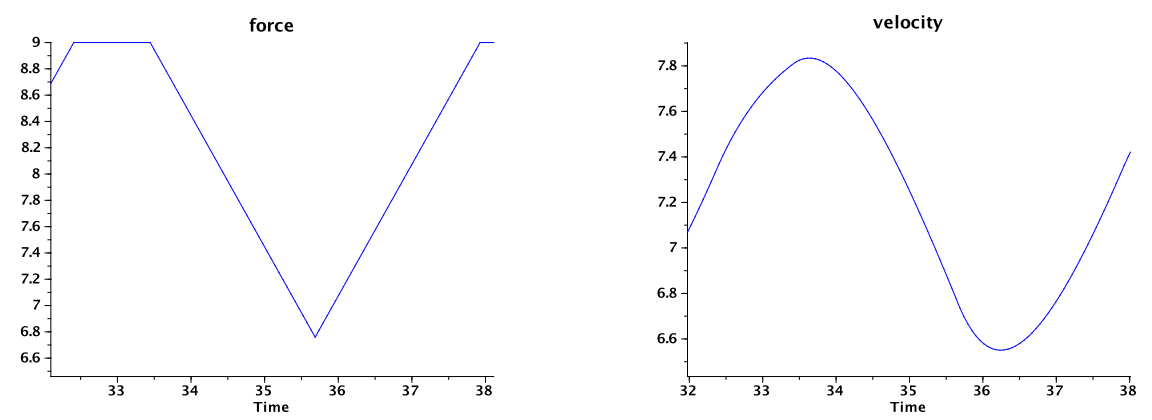

FIG. 2.5. Zoom of the case with recreation when slowing down (2000 time steps)
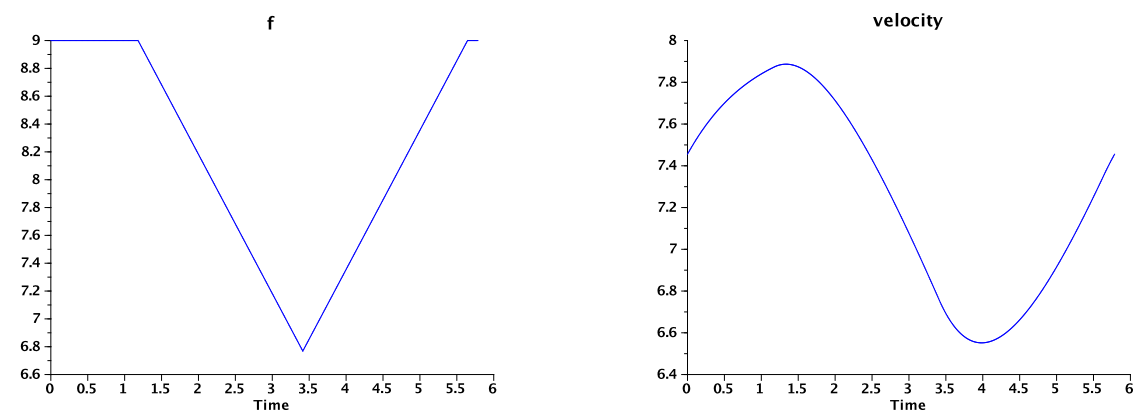

FiG. 2.6. Optimization over a period

This leads us to consider the problem of maximizing the average speed over a pe$\operatorname{riod} T$ (the period itself being an optimization parameter): the periodicity conditions apply to the speed and force, and the energy is such that $e(0)=e(T)+T e_{d}$. In other words, we wish to solve the following optimal control problem:

$$
\left\{\begin{aligned}
\operatorname{Min}-\frac{1}{T} \int_{0}^{T} v(t) \mathrm{d} t ; & v(0)=v(T) ; \quad e(0)=e(T)+T e_{d} ; \quad f(0)=f(T) . \\
& \dot{v}(t)=f(t)-\phi(t) ; \dot{e}(t)=\sigma+\eta(a(t))-f(t) v(t) ; \\
& |\dot{f}(t)| \leq 1, \quad \text { for a.e. } t \in(0, T) .
\end{aligned}\right.
$$

We can fix the initial energy to 0 .

Now we can compare figure 2.5 where we made a zoom on the solution computed before over the time interval $[32,38]$, with the solution of the periodic problem, displayed in figure 2.6. with period 5.79. We observe a good agreement between those two figures, which indicates that computing over a period may give a good approximation of the optimal trajectory.

3. Mathematical analysis. We have to study optimal control problems with a scalar state constraint and a scalar control, that in some of the models enters linearly in the state equation. We mention among others the related theoretical studies by Bonnans and Hermant [4] about state constrained problems, Maurer [14, who considers problems with bounded state variables and control appearing linearly, 
Felgenhauer 9] about the stability of singular arcs, and the two recent books by Osmolovskii and Maurer [19] and Schättler and Ledzewicz [21].

3.1. Statement of the model. we consider the following state equation

$$
\dot{h}=v ; \quad \dot{v}=f-\phi(v) ; \quad \dot{e}=\sigma(e)-f v,
$$

where the drag function $\phi$ satisfies

$$
\phi \text { is a } C^{2} \text { function; } \phi(0)=0, \phi^{\prime} \text { positive, } v \phi^{\prime}(v) \text { nondecreasing. }
$$

Since $\phi^{\prime}(v)>\phi^{\prime}(1) / v$, it follows that $\phi(v) \geq \phi(1)+\phi^{\prime}(1) \log v$ for all $v>0$, and so,

$$
\phi(v) \uparrow+\infty \text { when } v \uparrow+\infty \text {. }
$$

We assume for the moment (in section 3.3 we will discuss a more general recreation model) that the recreation function $\sigma(e)$ satisfies

$$
\sigma(e) \text { is } C^{2} \text { and nonnegative. }
$$

We will frequently mention Keller's model that correspond to the case when

$$
\phi(v)=v / \tau \text { and } \sigma(e) \text { is a positive constant. }
$$

As before, the initial condition is

$$
h(0)=0 ; \quad v(0)=0 ; \quad e(0)=e^{0}>0,
$$

and the constraints are

$$
0 \leq f(t) \leq f_{M} ; \quad e(t) \geq 0 ; \quad t \in(0, T) ; \quad-d(T) \leq-D .
$$

The problem is to minimize the time $T$ needed for reaching the final distance $d(T)=$ D.

The optimal control theory is introduced and discussed in Appendix D

3.2. Main results. If $0 \leq a<b \leq T$ is such that $e(t)=0$ for $t \in[a, b]$, but $e(\cdot)$ does not vanish over an interval in which $[a, b]$ is strictly included, then we say that $(a, b)$ is an arc with zero energy. Similarly, if $f(t)=f_{M}$ a.e. over $(a, b)$ but not over an open interval strictly containing $(a, b)$, we say that $(a, b)$ is an arc with maximal force. We define in a similar way arc with zero force, etc. We say that $t_{a}$ (resp. $t_{b}$ ) is the entry (resp. exit) time of the arc.

If the distance is small enough, then the strategy consists in setting the force to its maximal value. Let $D_{M}>0$ be the supremum (assumed to be finite) of the distance for which this property holds. using standard arguments based on minimizing sequences and weak topology (based on the fact that the control enters linearly in the state equation, the following can be easily proved:

LEMMA 3.1. The above problem has at least one optimal solution.

Theorem 3.2. Assume that $D>D_{M}$. Then: (i) An optimal strajectory starts with a maximal force arc, and is such that $e(T)=0$. (ii) If $\sigma$ is a positive constant, an optimal trajectory has the following structure: a maximal force arc, followed or not by a singular arc, and a zero energy arc.

This will be a consequence of Theorem 3.16 and Remark 3.3

Since, by proposition A.1, the optimal solutions are solutions of problems of maximizing the achieved distance in a given time, we consider in the sequel problems with a given final time. 


\subsection{Recreation when decreasing the speed.}

3.3.1. Framework. We next consider a variant of the previous model where the dynamics of the energy is a sum of functions of the energy and the acceleration, so that the dynamics are as follow:

$$
\left\{\begin{array}{l}
\dot{v}(t)=f(t)-\phi(v(t)) \\
\dot{e}(t)=\sigma(e(t))+\eta(f(t)-\phi(v(t)))-f(t) v(t) ;
\end{array}\right.
$$

with initial conditions

$$
v(0)=0 ; \quad e(0)=e^{0}>0,
$$

The constraints are as follows:

$$
0 \leq f(t) \leq f_{M} ; \quad e(t) \geq 0 ; \quad t \in(0, T) .
$$

The recreation optimal control problem is as follows:

$$
\operatorname{Min}-\int_{0}^{T} v(t) \mathrm{d} t ; \quad \text { s.t. } 3.8-3.10 .
$$

Denoting the acceleration by $a:=f-\phi(v)$, we may rewrite the above dynamics, skipping the time argument, as

$$
\left\{\begin{aligned}
\dot{v} & =a \\
\dot{e} & =\sigma(e)+\eta(a)-f v
\end{aligned}\right.
$$

We will assume that

$$
\eta \text { is a convex and } C^{1} \text { function, that vanishes over } \mathbb{R}_{+} .
$$

This implies that $\eta$ in nonincreasing.

A typical example is $\eta(a)=c\left|a_{-}\right|^{\beta}$, with $c \geq 0, \beta \geq 1$, and $a_{-}:=\min (a, 0)$. Let us denote the recovery obtained with a zero force (note that this is a $C^{1}$ and nondecreasing function with value 0 at 0 ) by

$$
R(v):=\eta(-\phi(v))
$$

and set

$$
\begin{aligned}
Q(v) & :=v \phi^{\prime}(v)+\phi(v)-R^{\prime}(v)\left(1-\phi(v) / f_{M}\right)+\phi^{\prime}(v) R(v) / f_{M} . \\
& =(v \phi(v))^{\prime}+(\phi(v) R(v))^{\prime} / f_{M}-R^{\prime}(v) .
\end{aligned}
$$

We will assume that (we can do this hypothesis later)

$$
\eta=c \bar{\eta}, \text { with } c \geq 0, \bar{\eta}(s)>0 \text { for all } s>0,
$$

and that $c$ and $\bar{\eta}$ are such that

$$
Q \text { is an increasing function of } v \text {. }
$$

REMARK 3.3. Assume that $\phi(v)=c_{\alpha} v^{\alpha}$ for some $c_{\alpha}>0$ and $\alpha \geq 1$, and that

$$
\bar{\eta}(a)=(-a)^{\beta}, \quad \text { for all } a<0 .
$$


Then $R(v)=c c_{\alpha}^{\beta} v^{\alpha \beta}$, and so

$$
Q(v)=\frac{\mathrm{d}}{\mathrm{d} v}\left(c_{\alpha} v^{\alpha+1}+c \frac{c_{\alpha}^{\beta+1}}{f_{M}} v^{\alpha \beta+\alpha}-c c_{\alpha}^{\beta} v^{\alpha \beta}\right)
$$

is positive and increasing for small enough $c$ i.

$$
\alpha+1 \leq \alpha \beta \text {, i.e., } 1+\alpha^{-1} \leq \beta .
$$

For instance, in Keller's model, $\alpha=1$ and the above condition holds iff $\beta \geq 2$.

Note that this holds when $\eta$ vanishes. An optimal control does not necessarily exist as the following theorem shows:

TheOREm 3.4. Let (3.16) hold with $c>0, \sigma(\cdot)$ be a positive constant, and (3.17) hold. Then no optimal control problem exists.

This will be proved at the end of the section, as a consequence of the analysis of the relaxed problem that we now perform. The theorem motivates the introduction of a relaxed problem.

3.3.2. Relaxed problem. In the relaxed formulation for which we refer to $[8$, we replace the control $f(t)$ with a probability distribution $\Xi(t, f)$ with value in $\left[0, f_{M}\right]$. Denoting by $\mathbb{E}_{\Xi(t)}$ the expectation associated with this probability measure, and by $\bar{\Xi}(t)$ the expectation of $f$ at time $t$, the state equation becomes

$$
\left\{\begin{array}{l}
\dot{v}(t)=\Xi(t)-\phi(v(t)), \\
\dot{e}(t)=\sigma(e(t))-\bar{\Xi}(t) v(t)+\mathbb{E}_{\Xi(t)} \eta(f-\phi(v(t))) .
\end{array}\right.
$$

The relaxed optimal control problem is

$$
\text { Min }-\int_{0}^{T} v(t) \mathrm{d} t ; \quad 3.21 \text { and } 3.9-3.10 \text { hold. }
$$

The Hamiltonian function is the same as for the non relaxed version, and its expression is

$$
H[p](f, v, e):=-v+p_{v}(f-\phi(v))+p_{e}(\sigma(e)-f v+\eta(f-\phi(v)) .
$$

The costate equation is therefore, omitting time arguments:

$$
\begin{cases}-\dot{p}_{v} & =-1-p_{v} \phi^{\prime}(v)-p_{e} \bar{\Xi}-p_{e} \phi^{\prime}(v) \mathbb{E}_{\Xi(t)} \eta^{\prime}(f-\phi(v)), \\ -\mathrm{d} p_{e} & =p_{e} \sigma^{\prime}(e) \mathrm{d} t-\mathrm{d} \mu .\end{cases}
$$

By standard arguments based on minimizing sequences we obtain that

LEMma 3.5. For $c \geq 0$ small enough, the above relaxed optimal control problem has at least one solution.

By lemma D.2 any feasible point of the above relaxed optimal control problem is qualified.

LEMma 3.6. For $c \geq 0$ small enough, the above relaxed optimal control problem has the same value as the original one, and therefore, any solution of the original problem is solution of the relaxed one.

Proof. Let $\Xi$ be a feasible point of the relaxed problem. For $c \geq 0$ small enough, by lemma D.2. for any $\varepsilon>0$, there exists a feasible point $\Xi^{\prime}$ of the relaxed problem

\footnotetext{
*We use the fact that over the compact set $\left[0, v_{M}\right]$, the fonction $\sum_{\gamma} v^{\delta}+c_{\gamma} v^{\gamma}$, with $0<\delta \leq \min \gamma$, is positive and increasing iff (given the $\gamma$ ) the $c_{\gamma}$ are small enough.
} 
such that $\left\|\Xi^{\prime}-\Xi\right\| \leq \varepsilon$ in the norm of $L^{\infty}(0, T, M([0, T]))$, with associated state $\left(v^{\prime}, e^{\prime}\right)$ which can be approximated [8] by a classical control $f$ whose state $\left(v^{f}, e^{f}\right)$ satisfies in the uniform norm $\left\|v^{f}-v^{\prime}\right\|+\left\|e^{f}-e^{\prime}\right\| \leq \varepsilon_{f}$, for arbitrary $\varepsilon_{f}>0$. When $\varepsilon_{f} \downarrow 0$ we have that $f$ is feasible for the (unrelaxed) optimal control problem, and the associated cost converges to the one associated with $\Xi^{\prime}$. The conclusion follows.

We have then that Pontryagin's principle holds in qualified form, i.e., with each optimal trajectory $(f, v, e)$ is associated at least one multiplier $(p, \mu)$ such that the relaxed control minimizes the Hamiltonian, in the sense that

$\Xi(t, \cdot)$ has support in $\operatorname{argmin}\left\{H[p(t)](f, v(t), e(t)) ; f \in\left[0, f_{M}\right]\right\}$, for a.a. $t$.

LEMMA 3.7. Let $t_{1} \in(0, T]$ be such that $e\left(t_{1}\right)=0$. Then: (i) we have that

$$
v\left(t_{1}\right) \geq \sigma(0) / f_{M} .
$$

(ii) An arc of maximal force cannot start at time $t_{1}$, or include time $t_{1}$.

Proof. (i) If $v\left(t_{1}\right)<\sigma(0) / f_{M}$, then for some $\varepsilon>0, \sigma(0)-v\left(t_{1}\right) f_{M}>2 \varepsilon$, so that for $t_{0} \in\left(0, t_{1}\right)$ close enough to $t_{1}$, since $\eta$ is nonnegative, we have that

$$
\dot{e}(t) \geq \sigma(e(t))-f(t) v(t) \geq \sigma\left(e\left(t_{1}\right)\right)-f_{M} v\left(t_{1}\right)-\varepsilon>\varepsilon>0,
$$

and so $e\left(t_{1}\right)=e\left(t_{0}\right)+\int_{t_{0}}^{t_{1}} \dot{e}(t) \mathrm{d} t>0$, which is a contradiction.

(ii) Let $t \in(0, T)$ be an entry point of a maximal force arc. Since $\eta$ vanishes on $\mathbb{R}_{+}$, and $f_{M} \geq \phi(v(\cdot))$, along the trajectory, we have $0 \leq \dot{e}\left(t_{+}\right)=\sigma(0)-f_{M} v(t)$. By point (i) this is an equality, and so $\dot{e}\left(t_{+}\right)=0$, and

$$
\ddot{e}\left(t_{+}\right)=\sigma^{\prime}(0) \dot{e}\left(t_{+}\right)-f_{M} \dot{v}\left(t_{+}\right)=-f_{M} \dot{v}\left(t_{+}\right)<0,
$$

implying that the energy cannot be positive after time $t$. This gives the desired contradiction.

LEMma 3.8. We have that (i) $T \in \operatorname{supp}(\mu)$, so that $e(T)=0$, and

$$
\left\{\begin{array}{lll}
\text { (ii) } & p_{e}(t)<0 & t \in[0, T) . \\
\text { (iii) } & p_{v}(t)<0 & t \in[0, T) .
\end{array}\right.
$$

Proof. (i) If $T \notin \operatorname{supp}(\mu)$, let $t_{e}:=\max \operatorname{supp}(\mu)$. Then $t_{e} \in(0, T)$, and $e\left(t_{e}\right)=0$. We analyze what happens over $\left(t_{e}, T\right)$. Since $p_{e}$ has derivative $\dot{p}_{e}=-p_{e} \sigma^{\prime}(e(t))$ and is continuous with zero value at time $T$, it vanishes, and so, $\dot{p}_{v}=1+p_{v} \phi^{\prime}(v)$. Since $p_{v}(T)=0$ this implies that $p_{v}$ has negative values. As $p_{e}$ vanishes and $p_{v}$ is negative, the Hamiltonian equal to $-v+p_{v}\left(f-\phi(v)\right.$ has a unique minimum at $f_{M}$. It follows that $\left(t_{e}, T\right)$ is included in a maximal force arc, which since $e\left(t_{e}\right)=0$ is in contradiction with lemma 3.7(ii). Point (i) follows.

(ii) If $p_{e}\left(t_{a}\right) \geq 0$ for some $t_{a} \in(0, T)$, then, by the costate equation, $p_{e}$ should vanish on $\left(t_{a}, T\right]$ and so $\left(t_{a}, T\right)$ would not belong to the support of $\mu$, in contradiction with (i). This proves (ii).

(iii) Let on the contrary $t_{c} \in[0, T)$ be such that $p_{v}\left(t_{c}\right) \geq 0$. Then the Hamiltonian is a sum of nondecreasing functions of $f$ and has a unique minimum point at 0 . Therefore $t_{c}$ belongs to a zero force arc, along which

$$
\dot{p}_{v}=1+p_{v} \phi^{\prime}(v)+p_{e} \eta^{\prime}(-\phi(v)) \phi^{\prime}(v)
$$


remains positive (remember that $p_{e}(t)<0$ and that $\eta$ is nonincreasing), and so this arc cannot end before time $T$. But then we cannot meet the final condition $p_{v}(T)=0$. The conclusion follows.

Since $p_{e}(t)<0$ over $(0, T)$, we deduce by 3.25 that if $(3.16)$ holds with $c>0$, then $H$ is a concave function of $f$ with minima in $\left\{0, f_{M}\right\}$ for a.a. $t$, and so, by (3.25):

Corollary 3.9. If (3.16) holds with $c>0$, then an optimal relaxed control has support over $\left\{0, f_{M}\right\}$ for a.a. $t$.

3.3.3. Reformulation of the relaxed problem. The previous corollary motivates the study of the case when we restrict the relaxed control to those having values in $\left\{0, f_{M}\right\}$. These relaxed control can be parameterized by their expectation $f(t)$ at any time $t$ : the probability to take the value 0 is $1-f(t) / f_{M}$. Remembering the definition of the recovery obtained with a zero force in (3.14), the dynamics can now be written in the form, since $\eta\left(f_{M}-\phi(v)\right)=0$ along the trajectory:

$$
\left\{\begin{array}{l}
\dot{v}(t)=f(t)-\phi(v(t)), \\
\dot{e}(t)=\sigma(e(t))-f(t) v(t)+\left(1-\frac{f(t)}{f_{M}}\right) R(v(t)),
\end{array}\right.
$$

with initial conditions 3.9 . The optimal control problem is

$$
\operatorname{Min}-\int_{0}^{T} v(t) \mathrm{d} t ; \quad 3.31 \text { and } 3.9 \text { hold, and } 0 \leq f \leq f_{M} \text { a.e., } e \geq 0 \text { on }[0, T] \text {. }
$$

REMARK 3.10. When $\eta$ identically vanishes, the above problem still makes sense and coincides with the formulation of the original model of section 3.1. So we will be able to apply the results of this section to Keller's problem.

The Hamiltonian is

$$
H^{R}=-v+p_{v}(f-\phi(v))+p_{e}(\sigma(e)-f v)+p_{e}\left(1-\frac{f}{f_{M}}\right) R(v) .
$$

The costate equations are

$$
\left\{\begin{aligned}
-\dot{p}_{v} & =-1-p_{v} \phi^{\prime}(v)-p_{e} f+p_{e}\left(1-\frac{f}{f_{M}}\right) R^{\prime}(v), \\
-\mathrm{d} p_{e} & =p_{e} \sigma^{\prime}(e) \mathrm{d} t-\mathrm{d} \mu
\end{aligned}\right.
$$

Of course we recover the expressions obtained in section 3.3 .2 in the particular case of relaxed controls with support in $\left\{0, f_{M}\right\}$, and therefore all lemmas of this section are still valid. By construction the Hamiltonian is an affine function of the control $f$, and the switching function is

$$
\Psi^{R}=H_{f}^{R}=p_{v}-p_{e}\left(v+R(v) / f_{M}\right) .
$$

Lemma 3.11. Any optimal trajectory starts with a maximal force arc.

Proof. Since $v(0)=0$, we have that $\Psi^{R}(0)=p_{v}(0)$ is negative by lemma 3.8(iii). We conclude with the PMP.

The following hypothesis implies that the energy is nonzero along a zero force arc.

Either $\sigma(0)>0$, or $\eta$ is nonzero over $\mathbb{R}^{-}$. 
Lemma 3.12. Let (3.36 hold. Then the jump of $\mu$ is equal to 0 on $[0, T]$.

Proof. a) Let $t \in[0, T)$ be such that $[\mu(t)]>0$. Necessarily $e(t)=0$, and so $t \in(0, T)$, and $\Psi^{R}\left(t_{-}\right) \leq 0$ (since otherwise $t$ belongs to a zero force arc and then by 3.36 we cannot have $e(t)=0)$. We have that $\left[p_{e}(t)\right]=[\mu(t)]>0$, and so $\left[\Psi^{R}(t)\right]=-\left(v(t)+R(v) / f_{M}\right)\left[p_{e}(t)\right]<0$, implying $\Psi^{R}\left(t_{+}\right)<0$. Therefore, for some $\varepsilon>0,(t, t+\varepsilon)$ is included in a maximal force arc, in contradiction with lemma 3.7 (ii).

b) If $[\mu(T)] \neq 0$, since $\lim p_{v}(t)=0$ and $\lim p_{e}(t)=-[\mu(T)]$ when $t \rightarrow T$, and $v(T)>0$, we get $\lim _{t \uparrow T} \Psi^{R}(t)=[\mu(T)]\left(v(T)+R(T) / f_{M}\right)>0$, meaning that the trajectory ends with a zero force arc, but then by $(3.36)$, the energy cannot vanish at the final time, contradicting lemma 3.8 (i).

By lemma 3.12 the switching function is continuous. When the state constraint is not active, its derivative satisfies

$$
\begin{aligned}
\dot{\Psi}^{R}= & 1+p_{v} \phi^{\prime}(v)+p_{e} f-p_{e}\left(1-\frac{f}{f_{M}}\right) R^{\prime}(v) \\
& +p_{e} \sigma^{\prime}(e)\left(v+R(v) / f_{M}\right)-p_{e}\left(1+R^{\prime}(v) / f_{M}\right)(f-\phi(v)) .
\end{aligned}
$$

The coefficient of $f$ cancel as expected and we find that, separating the contribution of $R(\cdot)$ :

$$
\begin{aligned}
\dot{\Psi}^{R}= & 1+p_{v} \phi^{\prime}(v)+p_{e}\left(\phi(v)+\sigma^{\prime}(e) v\right) \\
& +p_{e} \sigma^{\prime}(e) R(v) / f_{M}-p_{e} R^{\prime}(v)\left(1-\phi(v) / f_{M}\right)
\end{aligned}
$$

Subtracting $\Psi^{R} \phi^{\prime}(v)$ in order to cancel the coefficient of $p_{v}$, we obtain that

$$
\dot{\Psi}^{R}-\Psi^{R} \phi^{\prime}(v)=1+p_{e} \sigma^{\prime}(e)\left(v+R(v) / f_{M}\right)+p_{e} Q(v),
$$

where $Q$ was defined in 3.15 .

LEMмA 3.13. For given $0 \leq t_{1}<t_{2} \leq T$, Assume that $\left(t_{1}, t_{2}\right)$ is included in a singular arc over which $\sigma$ is constant. Then, over $\left(t_{1}, t_{2}\right), Q(v)$ is constant and, if the function $Q$ is not constant on any interval, $v$ is constant.

Proof. Along a singular arc, $\mathrm{d} \mu$ vanishes, so that if $\sigma$ is constant, so is $p_{e}$. By 3.39, so is also $Q(v)=-1 / p_{e}$. The conclusion follows.

REMARK 3.14. If $\eta$ is analytic over $\mathbb{R}_{-}$and $\phi$ is analytic over $\mathbb{R}_{+}$, then $Q$ is analytic over $\mathbb{R}_{+}$, and therefore is either constant over $\mathbb{R}$, or not constant on any interval of $\mathbb{R}_{+}$.

We say that $\bar{t} \in(0, T)$ is a critical time if $\Psi(t)=0$, and we say that $\bar{t}_{ \pm}$is energy free if $e(t)>0$ for $t$ in $(\bar{t}, \bar{t} \pm \varepsilon)$, for $\varepsilon>0$ small enough. As for 3.39 , we have that, for such times the existence of left or right derivatives:

$$
\dot{\Psi}_{t \pm}^{R}=1+p_{e} \sigma^{\prime}(e)\left(v+R(v) / f_{M}\right)+p_{e} Q(v),
$$

where $Q(\cdot)$ was defined in 3.40 . We now need to assume that 3.17 holds.

LEMMA 3.15. Let $\sigma$ be a positive constant, and (3.17) hold. Then, along an optimal trajectory: (i) There is no zero force arc, and hence, $\Psi(t) \leq 0$, for all $t \in$ $[0, T]$. (ii) The only maximal force arc is the one starting at time 0 .

Proof. (i) Let $\left(t_{a}, t_{b}\right)$ be a zero force arc, over which necessarily $\Psi$ is nonnegative. By lemma 3.11, $t_{a}>0$, and so $\Psi\left(t_{a}\right)=0$ and $\dot{\Psi}\left(t_{a+}\right) \geq 0$. Since $\sigma$ is constant and positive, over the arc, $e(t)>0, p_{e}$ is constant, the speed is decreasing, and so by 3.40 , we have that $\dot{\Psi}\left(t_{b-}\right)>\dot{\Psi}\left(t_{a+}\right) \geq 0$ meaning that the zero force arc cannot end 

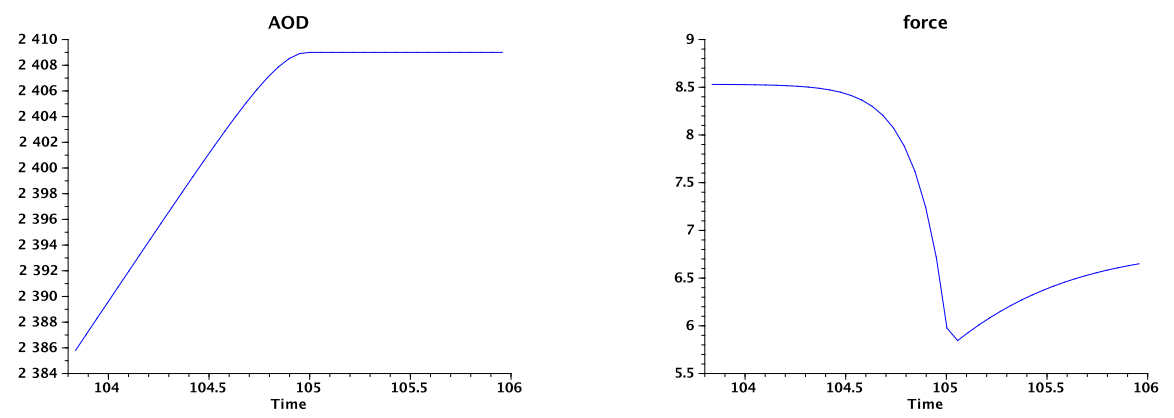

FIG. 3.1. Race problem with Keller's model: zoom on end of race: AOD and force.

before time $T$, contradicting the final condition $e(T)=0$.

(ii) On a maximal force arc $\left(t_{a}, t_{b}\right)$ with $t_{a}>0$, since the speed increases, (3.40) implies $\dot{\Psi}\left(t_{b-}\right)<\dot{\Psi}\left(t_{a+}\right) \leq 0$, and since $\Psi_{t} \leq 0$ along the maximal force arec, it follows that $\Psi\left(t_{b}\right)<0$, meaning that the maximal force arc ends at time $T$. But then $\left[p_{e}(T)\right]=[\mu(T)]>0$, in contradiction with lemma 3.12 .

THEOREM 3.16. Let $\sigma$ be a positive constant, and (3.17) hold. Then an optimal trajectory has the following structure: maximal force arc, followed or not by a singular arc, and a zero energy arc.

Proof. The existence of a maximal force arc starting at time 0 is established in lemma 3.11. Let $t_{a} \in(0, T)$ be its exit point $\left(t_{a}=T\right.$ is not possible since $\left.T>T_{M}\right)$, and let $t_{b} \in(0, T)$ be the first time at which the energy vanishes (that $t_{b}<T$ follows from lemmas 3.8(i) and 3.12). If $t_{a}<t_{b}$, over $\left(t_{a}, t_{b}\right)$, by lemma 3.15, $\Psi$ is equal to zero and hence, $\left(t_{a}, t_{b}\right)$ is a singular arc. Finally let us show that the energy is zero on $\left(t_{b}, T\right)$. Otherwise there would exist $t_{c}, t_{d}$ with $t_{b} \leq t_{c}<t_{d} \leq T$ such that $e\left(t_{c}\right)=e\left(t_{d}\right)=0$, and $e(t)>0$, for all $t \in\left(t_{c}, t_{d}\right)$. By lemma 3.15, $\left(t_{c}, t_{d}\right)$ is a singular arc, over which $\dot{e}=\sigma-f v$ is constant. which gives a contradiction since the energy varies along this arc. The result follows.

Proof. [Proof of theorem 3.4 By lemma 3.6, any solution of the classical problem is solution of the relaxed one. By theorem 3.16 the trajectory must finish with an arc of zero energy, over which $0=\dot{e}(t)=\sigma-f(t) v(t)+\eta(f(t)-\phi(v(t))$. The r.h.s. is a decreasing function of $f(t)$. We deduce that $f(t)$ is a continuous function of $v(t)$, and hence, of time over this arc. On the other hand, since $p_{e}<0$ a.a., the Hamiltonian is a concave function of $f$ which is not affine on $\left[0, f_{M}\right]$, and so attains its minima at either 0 or $f_{M}$. Therefore $f(t)$ is constant and equal to either 0 or $f_{M}$ over the zero energy arc. For $f(t)=0$ we have that $\dot{e}(t)$ is positive. That $f(t)=f_{M}$ is not possible since we know that the trajectory has only one maximal force arc. We have obtained the desired conclusion.

REMARK 3.17. We plot in figure 3.1 a zoom on the end of the race. While we have proved that for the continuous problem, there is a switching time from the singular arc, with constant speed, to the zero energy arc, we observe in the discretized problem a progressive transition between 104 and 105 seconds.

3.4. Bounding variations of the force. It seems desirable to avoid discontinuities of the force that occur with the previous model, and for that we introduce bounds on $\dot{f}$. The force becomes then a state and the new control $\dot{f}$ is denoted by $g$. 
So the state equation is (note that we have taken here $\sigma=0$ )

$$
\dot{v}=f-\phi(v) ; \quad \dot{e}=\sigma(e)+\eta(a)-f v ; \quad \dot{f}=g,
$$

with constraints

$$
0 \leq f \leq f_{M} ; \quad e \geq 0 ; \quad g_{m} \leq g \leq g_{M}
$$

We minimize as before $-\int_{0}^{T} v(t) \mathrm{d} t$. The Hamiltonian is

$$
H=-v+p_{v}(f-\phi(v))+p_{e}(\eta(f-\phi(v))-f v)+p_{f} g .
$$

The costate equation $-\dot{p}=H_{y}$ are now

$$
\begin{cases}-\dot{p}_{v} & =-1-p_{v} \phi^{\prime}(v)-p_{e}\left(\eta^{\prime}(a) \phi^{\prime}(v)+f\right), \\ -\mathrm{d} p_{e} & =\sigma^{\prime}(e) p_{e} \mathrm{~d} t-\mathrm{d} \mu \\ -\dot{p}_{f} & =p_{v}+p_{e}\left(\eta^{\prime}(a)-v\right) .\end{cases}
$$

The state constraint $e \geq 0$ is of second order, and we may expect a jump of the measure $\mu$ at time $T$. The final condition for the costate are therefore

$$
p_{v}(T)=0 ; \quad p_{e}(T)=0 ; \quad p_{f}(T)=0 .
$$

We may expect and will assume that the above two inequalities are strict. By the analysis of the previous section we may expect that the optimal trajectory is such that $g$ is bang-bang (i.e., always on its bounds).

4. Conclusion. We have established a system of ordinary differential equations governing the evolution of the velocity $v$, the anaerobic energy $e_{a n}$, and the propulsive force $f$. This is based on the equation of motion (relating the acceleration $a=d v / d t$ to the propulsive force and the resistive force) and a balance of energy. Several constraints have to be taken into account: the propulsive force is positive and less than a maximal value, its derivative has to be bounded, the anaerobic energy is positive. Keller [11, 12] used in his model the evolution of the aerobic energy, which is not satisfactory. Here, using a hydraulic analogy initiated by Morton [15, 16], we manage to write an equation for the instantaneous accumulated oxygen deficit instead. In our model, in difference with respect to Keller's, we introduce variations in $\sigma$, modeling the oxygen uptake, $\dot{V} O 2$ : indeed, one of the roles of the anaerobic energy is to compensate the deficit in oxygen uptake, $\dot{V} O 2$, which has not reached its maximal value at the beginning of the race. Conversely, when the anaerobic energy gets too low, the oxygen uptake $\dot{V} O 2$ cannot be maintained to its maximal value. We make two further extensions: we introduce a physiological observation that energy is recreated when the acceleration is negative, that is when decreasing the speed, and the fact that the derivative of the propulsive force has to be bounded.

Our model could be used, in the simulations, given the velocity profile of a runner, to compute the evolution of his anaerobic energy. This is an important challenge for sportsmen to determine instantaneous anaerobic energy consumption.

In this paper, we use our system for the optimization of strategy in a race: given a distance, we want to find the optimal velocity leading to the shortest run. Our main results are illustrated in Figures 2.3, 2.4. Without the energy recreation term, we find that negative splitting of the race (running the second half quicker than the first half) is the best strategy. Our numerical simulations on the final model provide oscillations 
of the velocity and energy recreation that qualitatively reproduce the physiological measurements of $[3]$.

Using optimal control theory, we manage to get rigorous proofs of most of our observations. We prove in particular that in the case of Keller, the race is made up of exactly three parts: run at maximal propulsive force, run at constant speed (corresponding to a singular arc), run at zero energy. It cannot be made of any other arcs. For this purpose, we relate the problem to a relaxed formulation, where the propulsive force represents a probability distribution rather than a function of time. We also find that the concavity of the Hamiltonian results in speed oscillations and we show how, by reducing the problem on optimizing over a period, we recover the latter.

Appendix A. Abstract distance and time functions. In this section we establish in a general setting the relation between the distance and time functions, defined as above. Set

$$
\mathcal{U}_{T}:=L^{\infty}(0, T) ; \quad \mathcal{Y}_{T}:=L^{\infty}\left(0, T ; \mathbb{R}^{n}\right) .
$$

Given $\delta: \mathbb{R}^{n} \rightarrow \mathbb{R}, F: \mathbb{R} \times \mathbb{R}^{n} \rightarrow \mathbb{R}^{n}$, and $K_{T} \subset \mathcal{U}_{T} \times \mathcal{Y}_{T}$, we consider the "abstract" problems of minimal time

$\left(P_{D}\right) \operatorname{Min} T ; \dot{y}(t)=F(f(t), y(t)), t \in(0, T), y(0)=y^{0} ;(f, y) \in K_{T} ; \delta(y(T))=D$,

and of maximal distance

$\left(P_{T}\right) \quad \operatorname{Max} \delta(y(T)) ; \quad \dot{y}(t)=F(f(t), y(t)), t \in(0, T), y(0)=y^{0} ; \quad(f, y) \in K_{T}$.

In our examples $y=(h, v, e)$ and $\delta(y)=y_{1}$ is the distance.

Proposition A.1. Denote by $\mathcal{T}(D)$ and $\mathcal{D}(T)$ the optimal values of the above problems. Assume that (i) These functions are finitely valued, nondecreasing and continuous over $\mathbb{R}_{+}$with value 0 at 0 . (ii) any feasible trajectory $(\hat{f}, \hat{y})$ for $\left(P_{D}\right)$ with cost function $\hat{T}$ is feasible for $\left(P_{\hat{T}}\right)$ (ii) any feasible trajectory $(\bar{f}, \bar{y})$ for $\left(P_{T}\right)$ with cost function $\bar{D}$ is feasible for $\left(P_{\bar{D}}\right)$. Then (a) $T(D)$ is the inverse function of $D(T)$, and (b) any optimal solution of $\left(P_{T}\right)$ (resp. $\left.\left(P_{D}\right)\right)$ is solution of $\left(P_{\mathcal{D}(T)}\right)$ (resp. $\left(P_{\mathcal{T}(D)}\right)$ ).

Proof. (a1) Given $\varepsilon>0$, let $(\hat{f}, \hat{y})$ be as above and such that $\hat{T} \leq \mathcal{T}(D)+\varepsilon$. Then

$$
D \leq \mathcal{D}(\hat{T}) \leq \mathcal{D}(\mathcal{T}(D)+\varepsilon)
$$

The first inequality is due to the fact that the trajectory $(\hat{f}, \hat{y})$ is feasible for $\left(P_{\hat{T}}\right)$, and the second one holds since $\mathcal{D}$ is nondecreasing. Passing to the limit when $\varepsilon \downarrow 0$ and using the continuity of $\mathcal{D}$, we deduce that

$$
D \leq \mathcal{D}(\mathcal{T}(D))
$$

(a2) Given $\varepsilon>0$, let $(\bar{f}, \bar{y})$ be as above and such that $\mathcal{D}(T)-\varepsilon \leq \bar{D}$. Then

$$
\mathcal{T}(\mathcal{D}(T)-\varepsilon) \leq \mathcal{T}(\bar{D}) \leq T .
$$

The first inequality holds since $\mathcal{T}$ is nondecreasing, and the second one is due to the fact that the trajectory $(\bar{f}, \bar{y})$ is feasible for $\left(P_{\bar{D}}\right)$. Passing to the limit when $\varepsilon \downarrow 0$ and using the continuity of $\mathcal{T}$, we deduce that $\mathcal{T}(\mathcal{D}(T)) \leq T$. 
(a3) Combining with A.3 we get $\mathcal{T}(D) \leq \mathcal{T}(\mathcal{D}(\mathcal{T}(D))) \leq \mathcal{T}(D)$, so that for all $T=\mathcal{T}(D)$, we have that $T=\mathcal{T}(\mathcal{D}(T)$ ). Point (a) follows.

(b) Easy consequence of point (a).

Appendix B. Strategy of maximal force. The strategy of maximal force is the one for which the force always has its maximal value. Then speed is an increasing function of time, with positive derivative, and asymptotic value

$$
v_{M}=\phi^{-1}\left(f_{M}\right) \quad\left(v_{M}=\tau f_{M} \text { in Keller's model }\right) .
$$

Note that, by 3.2 - 3.3$), \phi^{-1}\left(f_{M}\right)$ is a locally Lipschitz function $\mathbb{R} \rightarrow \mathbb{R}$. So we have that

$$
v(t)<v_{M}, \dot{v}(t)>0, \text { and } v(t) \uparrow v_{M} \text { if } f(t)=f_{M} \text { for all } t \geq 0 .
$$

We first discuss the existence of a critical distance $D_{M}$ at which the energy vanishes.

Lemma B.1. (i) The energy cannot remain nonnegative for all time $t \geq 0$ if, for some $\varepsilon_{M}>0$,

$$
\sup _{e \geq 0} \sigma(e)<f_{M} v_{M}=f_{M} \phi^{-1}\left(f_{M}\right)
$$

(ii) If the energy vanishes at time $t_{M}$, then the maximal force strategy does not respect the contraint of nonnegative energy over $[0, t]$ for any $t>t_{M}$.

Proof. (i) By (B.3), there exists $\varepsilon_{M}>0$ such that $\left.\sup _{e \geq 0} \sigma(e)\right)+\varepsilon<f_{M} v_{M}$. For large enough time, $\dot{e}(t) \leq-\varepsilon_{M}$ so that $e(t) \rightarrow-\infty$; point (i) follows.

(ii) If the conclusion does not hold, then $e$ attains its minimum over $(0, \tau)$ at time $t_{M}$, and so we have $e\left(t_{M}\right)=0$ and $\dot{e}\left(t_{M}\right)=\sigma(0)-f_{M} v\left(t_{M}\right)=0$. Since the speed has a positive derivative, it follows that

$$
\ddot{e}\left(t_{M}\right)=\sigma^{\prime}(0) \dot{e}\left(t_{M}\right)-f_{M} \dot{v}\left(t_{M}\right)=-f_{M} \dot{v}\left(t_{M}\right)<0,
$$

and therefore in any case $e(t)<0$ for $t>t_{M}$, close to $t_{M}$, which gives the desired contradiction.

Appendix C. The Bellman function. More generally we denote the maximal distance one can run in time $T$ by $D\left(T, v^{0}, e^{0}\right)$, starting with initial condition $v_{0}=v^{0}$, $e_{0}=e^{0}$.

Lemma C.1. The function $D\left(T, v^{0}, e^{0}\right): \mathbb{R}_{+}^{3} \rightarrow \mathbb{R}$ is an increasing function of every of its three arguments.

Proof. That $D$ is a increasing function of $T$ is easy to prove. When changing the initial energy from $e^{0}$ to $\hat{e}^{0}>e^{0}$, given an optimal control and state $(\bar{f}, \bar{v}, \bar{e})$, we see that $\bar{f}$ is feasible for the new problem, since the state has the same speed $\bar{v}$ and a new energy $\hat{e} \geq \bar{e}$. It follows that $D$ is an increasing function of $e^{0}$. Finally, let us change the initial speed to $\hat{v}^{0}>v^{0}$. If, for a zero force strategy, the corresponding speed $\hat{v}$ is always greater that $\bar{v}$ over $(0, T)$, the conclusion holds. Otherwise, let $t_{a} \in(0, T)$ be such that when applying the zero force over $\left(0, t_{a}\right)$, we have that $\hat{v}\left(t_{a}\right)=v\left(t_{a}\right)$. Define the strategy $\hat{f}$ to have value 0 over $\left(0, t_{a}\right)$, and to be equal to $f$ otherwise.

Clearly, the distance at time $t_{a}$ is greater than the corresponding one for the original strategy, and the energy denoted by $\hat{e}$ satisfies $\hat{e}\left(t_{a}\right)>\bar{e}\left(t_{a}\right)$ (equality is not possible since it would mean that $\bar{f}(t)=0=\hat{f}(t)$ for all $t \in\left(0, t_{a}\right)$, but then 
$\left.\hat{v}\left(t_{a}\right)>\bar{v}\left(t_{a}\right)\right)$. Since we know that $D$ is an increasing function of energy), the conclusion follows.

Appendix D. Qualification. We consider the model with energy recreation of section 3.3. We assume that the functions $\sigma, \Phi$ and $\bar{\eta}$ are of class $C^{1}$. Set as before $\eta=c \bar{\eta}$ for some $c \geq 0$. The mapping $(v[f], e[f])$ is of class $C^{1}$ and the directional derivative in the direction $\delta f \in \mathcal{U}$ is solution of the linearized state equation, i.e.,

$$
\left\{\begin{aligned}
\dot{\delta} v(t)= & \delta f(t)-\phi^{\prime}(v(t)) \delta v(t), \quad t \geq 0 \\
\dot{\delta} e(t)= & \sigma^{\prime}(e(t)) \delta e(t)-\delta f(t) v(t)-f(t) \delta v(t) \\
& +\eta^{\prime}(a(t))\left(\delta f(t)-\phi^{\prime}(v(t) \delta v(t)) \quad t \geq 0\right. \\
0 & \delta v(0)=\delta e(0) .
\end{aligned}\right.
$$

We denote the solution of this system by $(\delta v[\delta f], \delta e[\delta f])$. Let us write the constraints in the form

$$
f \in \mathcal{U}_{M} \text { and } e[f] \in K
$$

where

$$
\mathcal{U}_{M}:=\left\{f \in \mathcal{U} ; \quad 0 \leq f(t) \leq f_{M} \text { a.e. }\right\} ; \quad K=C([0, T])_{+}
$$

Let $f \in \mathcal{U}_{M}$ be a feasible control, i.e., which is such that $f \in \mathcal{U}_{M}$ and $e[f] \in K$. The constraints are said to be qualified at $f$ (see [20] or [7, section 2.3.4]) if there exists $\delta f \in \mathcal{U}$ such that

$$
f+\delta f \in \mathcal{U}_{M} ; \quad e[f]+\delta e[\delta f] \in \operatorname{int}(K) .
$$

In other word, the variation $\delta f$ of the control is compatible with the control constraints, and the linearized state $\delta e$ allows to reach the interior of the set of feasible states. Remember that $e(0)>0$.

LEMmA D.1. If $c$ is small enough, the optimal control problem (3.11) is qualified.

Proof. a) We first obtain the result when $c=0$. If $e(t)$ is always positive the qualification holds with $\delta f=0$. Otherwise, let $t_{a}$ be the smaller time at which the energy vanishes. with $\delta f=-f$. Obviously $f+\delta f \in \mathcal{U}_{M}$, and since $\delta f$ is a.e. nonpositive, so is $\delta v$. Next, since $c=0$, we have that

$$
\dot{\delta} e(t)=\sigma^{\prime}(e(t)) \delta e(t)-\delta f(t) v(t)-f(t) \delta v(t), \quad t \in(0, T) ; \quad \delta e(0)=0,
$$

that implies $\delta e \geq 0$. Let $t_{b}$ be the essential supremum of times for with $f(t)$ is zero. Clearly, $t_{b}<t^{a}$, and for any $\varepsilon>0$, there exists $\alpha>0$ such that

$$
v(t) \geq \alpha \text { and }-\delta v(t) \geq \alpha, \text { for all } t \in\left[t_{b}+\varepsilon, T\right] .
$$

Then for $t$ in $\left(t^{f}+\varepsilon, T\right]$ :

$$
\begin{aligned}
\dot{\delta} e(t) & \left.\geq \sigma^{\prime}(e(t)) \delta e(t)-\left(f(t)+\eta^{\prime}(a(t)) \phi^{\prime}(v(t))\right) \delta v(t)\right) \\
& \geq-C \delta e(t)+2 \alpha f(t) .
\end{aligned}
$$

Taking $\varepsilon \in\left(0, t^{a}-t^{f}\right)$, it follows that $\delta e(t)>0$ over $\left[t^{a}, T\right]$, and so $e(t)+\delta e(t)$ is positive over $[0, T]$, and hence uniformly positive as was to be shown.

b) We now show that for $c>0$ small enough the qualification can be obtained, again by taking $\delta f=-f$. Given a sequence $c_{k}$ of positive number converging to 0 and 
$f_{k} \in \mathcal{U}_{M}$, we may extract a subsequence such that $f_{k}$ converges to $f$ in $L^{\infty}$ weak*, and since $c_{k} \downarrow 0$, the associated states $\left(v_{k}, e_{k}\right)$ uniformly converge to the associated state $(v, e)$. Since $\delta f_{k}$ converges to $\delta f$ in $L^{\infty}$ weak*, we deduce that $\left(\delta v_{k}, \delta e_{k}\right)$ uniformly converge to $(\delta v, \delta e)$. By point (a), $e_{k}+\delta e_{k}$ is (uniformly) positive over $[0, T]$, as was to be shown.

We now consider the relaxed formulation of section 3.3.2.

LEMma D.2. If $c$ is small enough, the optimal control problem (3.22) is qualified.

Proof. The proof is essentially the same, up to technical details (the main point is that for the variation of the control we still take the opposite of the control), and is left to the reader. $\square$

Acknowledgments. The first author would like to thank Christophe Clanet for pointing out the reference by Keller, Véronique Billat for discussions on the physiological models and the bibliography, and Nadège Arnaud who made figure 2.1. The second author acknowledges support from the EU 7th Framework Programme (FP7PEOPLE-2010-ITN), under GA number 264735-SADCO.

\section{REFERENCES}

[1] H. Behncke. A mathematical model for the force and energetics in competitive running. Journal of mathematical biology, 31(8):853-878, 1993.

[2] V. Billat. Physiologie et méthodologie de l'entraînement: De la théorie à la pratique. Sciences et pratiques du sport. De Boeck Université, 2012. Third edition.

[3] V. Billat, L. Hamard, J.P. Koralsztein, and R.H. Morton. Differential modeling of anaerobic and aerobic metabolism in the 800-m and 1,500-m run. J Appl Physiol., 107(2):478-87, 2009.

[4] J.F. Bonnans and A. Hermant. Second-order analysis for optimal control problems with pure state constraints and mixed control-state constraints. Ann. Inst. H. Poincaré Anal. Non Linéaire, 26(2):561-598, 2009.

[5] J.F. Bonnans and J. Laurent-Varin. Computation of order conditions for symplectic partitioned Runge-Kutta schemes with application to optimal control. Numer. Math., 103(1):1-10, 2006.

[6] J.F. Bonnans, P. Martinon, and V. Grélard. Bocop v1.0.3: A collection of examples. Url: www.bocop.org, June 2012.

[7] J.F. Bonnans and A. Shapiro. Perturbation analysis of optimization problems. Springer-Verlag, New York, 2000.

[8] I. Ekeland and R. Temam. Convex analysis and variational problems, volume 1 of Studies in Mathematics and its Applications. North-Holland, Amsterdam, 1976. French edition: Analyse convexe et problèmes variationnels, Dunod, Paris, 1974.

[9] U. Felgenhauer. Structural stability investigation of bang-singular-bang optimal controls. J. Optim. Theory Appl., 152(3):605-631, 2012.

[10] W.W. Hager. Runge-Kutta methods in optimal control and the transformed adjoint system. Numer. Math., 87(2):247-282, 2000.

[11] Joseph B. Keller. A theory of competitive running. Physics Today, 26(9):42-47, 1973.

[12] Joseph B. Keller. Optimal velocity in a race. Amer. Math. Monthly, 81:474-480, 1974.

[13] F. Mathis. The effect of fatigue on running strategies. SIAM Review, 31(2):306-309, 1989.

[14] H. Maurer. On optimal control problems with bounded state variables and control appearing linearly. SIAM J. Control Optimization, 15(3):345-362, 1977.

[15] R.H. Morton. A 3-parameter critical power model. Ergonomics, 39(4):611-619, 1996.

[16] R.H. Morton. The critical power and related whole-body bioenergetic models. European Journal of Applied Physiology, 96(4):339-354, 2006.

[17] R.H. Morton. A new modelling approach demonstrating the inability to make up for lost time in endurance running events. IMA Journal of Management Mathematics, 20(2):109-120, 2009. 
[18] R.H. Morton and V.Billat. The critical power model for intermittent exercise. European Journal of Applied Physiology, 91(2-3):303-307, 2004.

[19] N.P. Osmolovskii and H. Maurer. Applications to regular and bang-bang control, volume 24 of Advances in Design and Control. Society for Industrial and Applied Mathematics (SIAM), Philadelphia, PA, 2012. Second-order necessary and sufficient optimality conditions in calculus of variations and optimal control.

[20] S.M. Robinson. First order conditions for general nonlinear optimization. SIAM Journal on Applied Mathematics, 30:597-607, 1976.

[21] H. Schättler and U. Ledzewicz. Geometric optimal control, volume 38 of Interdisciplinary Applied Mathematics. Springer, New York, 2012. Theory, methods and examples.

[22] Andreas Wächter and Lorenz T. Biegler. On the implementation of an interior-point filter line-search algorithm for large-scale nonlinear programming. Math. Program., 106(1, Ser. A):25-57, 2006.

[23] A.J. Ward-Smith. A mathematical theory of running, based on the first law of thermodynamics, and its application to the performance of world-class athletes. Journal of Biomechanics, 18(5):337 - 349, 1985.

[24] W. Woodside. The optimal strategy for running a race (a mathematical model for world records from $50 \mathrm{~m}$ to $275 \mathrm{~km})$. Math. Comput. Modelling, 15(10):1-12, 1991. 